\title{
Ein neues, unkompliziert auszuführendes Verfahren zur Bestimmung kleiner Konzentrationen an Wasser in organischen Lösungsmitteln
}

\author{
Heinz Langhals \\ Chem. Lab. der Univ. Freiburg, Albertstr. 21, D-7800 Freiburg i. Br.
}

\section{A New, Uncomplicated Procedure \\ for the Determination of Small Concentrations of Water in Organic Solvents}

Summary. A new procedure for the determination of water (even in trace amounts) in organic solvents is described. The solvatochromism of the pyridiniumphenol betaine, $E_{T} 30$, determined by a simple UVabsorption measurement, together with a twoparameter equation, permits an exact determination. The procedure is rapid and is, therefore, an alternative to the Karl-Fischer titration.

Zusammenfassung. Ein neues Verfahren zur Bestimmung von Wasser in organischen Lösungsmitteln (auch in Spuren) wird beschrieben. Die Solvatochromie des Pyridiniumphenolbetains $E_{T} 30$, die über eine einfach auszuführende UV-Absorptionsmessung bestimmt wird, ermöglicht zusammen mit einer zwei-ParameterGleichung eine exakte Wasserbestimmung in organischen Lösungsmitteln als Schnelltest und stellt damit eine Alternative zur Karl-Fischer-Titration dar.

Key words: Best. von Wasser in organ. Lösungsmitteln; Spektralphotometrie; Pyridiniumphenolbetain, 2Parameter-Gleichung

Die Bestimmung des Wassergehaltes organischer Lösungsmittel, welche beim Arbeiten mit feuchtigkeitsempfindlichen Reagentien, wie z. B. Metallorganylen oder Säurehalogeniden, notwendig ist, wird heute üblicherweise über die Karl-Fischer-Titration vorgenommen [8]. Die Bestimmungsmethode benötigt zur Titration wasserfreie Lösungsmittel und Reagentien und damit eine spezielle Bestimmungsapparatur. Ein direk- tes, visuelles Erkennen des Titrationsendpunktes ist schwierig. Die Titration wird daher üblicherweise mit einer speziellen Apparatur nach der »dead-stop-Methode« ausgeführt. Weitere Probleme können bei Verwendung älterer Titrationslösungen oder in Gegenwart von Redoxsystemen auftreten [8]. Die oben angegebenen Probleme legen nahe, für Routine-Untersuchungen ein einfacher auszuführendes Verfahren zu entwickeln.

Im vorliegenden Beitrag wird ein neues Verfahren beschrieben, mit dem der Wassergehalt von organischen Lösungsmitteln mit geringem Aufwand in einem Schnelltest exakt bestimmt werden kann. Bei diesem Schnelltest wird ausgenutzt, daß Wasser wesentlich polarer ist als fast alle organischen Lösungsmittel und deren übliche Verunreinigungen. Daher führt bereits ein geringer Wassergehalt zu einer erheblichen Polaritätssteigerung, während anderweitige Verunreinigungen in vergleichbarer Menge die Polarität nicht nennenswert beeinflussen. Auf diese Weise ist sehr genau eine Bestimmung des Wassergehaltes über eine Messung der Lösungsmittelpolarität möglich.

Für die Bestimmung wird die starke Solvatochromie des von Dimroth u. Reichardt [3] synthetisierten Pyridiniumphenolbetains $E_{T} 30$ (l) verwendet.

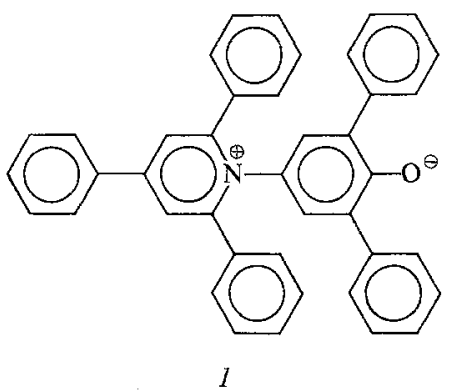

Mit diesem Farbstoff haben Dimroth $u$. Reichardt die Gemische Ethanol-Wasser, 2,6-Lutidin-Wasser [7], 
Tabelle 1. Konstanten von Gl. (1) für häufig verwendete Lösungsmittel

\begin{tabular}{lcrcc}
\hline Lösungsmittel & $E_{T}^{\mathrm{a} a \mathrm{~b}}$ & $c^{* \mathrm{c}}$ & $E_{D}^{\mathrm{a}}$ & $E_{T}^{0} / E_{D}$ \\
\hline Aceton & 42,4 & 0,31 & 2,83 & 14,9 \\
Acetonitril & 46,0 & 0,15 & 2,07 & 22,2 \\
tert.-Butylalkohol & 43,9 & 1,01 & 2,82 & 15,6 \\
tert.-Butylhydroperoxid $^{\text {Dimethylformamid }}$ & 49,7 & 0,31 & 1,40 & 35,5 \\
1,4-Dioxan & 43,8 & 11,43 & 9,24 & 4,74 \\
Pyridin $^{\mathrm{d}}$ & 36,0 & 0,58 & 4,34 & 8,29 \\
& 40,2 & 5,48 & 7,09 & 5,67 \\
\hline
\end{tabular}

a In $\mathrm{kcal} \cdot \mathrm{Mol}^{-1}$

b Siehe auch Lit. [6]

- In $\mathrm{Mol} \cdot 1^{-1}$

d Bei älteren, peroxidreichen Proben, die 1 zerstören, empfiehlt sich der Zusatz einer Base z. B. Piperidin

Aceton-Wasser [3], Pyridin-Wasser [3], Methanol-Wasser, $i$-Propylalkohol-Wasser, Piperidin-Wasser und Dioxan-Wasser [2] untersucht. Die von ihnen erhaltenen Kurven $\left(E_{T} 30\right.$ als Funktion der Zusammensetzung in Vol- $\%$ ) sind jedoch über den gesamten gemessenen Bereich stark gekrümmt. Würde man versuchen, auf diesem Wege Wasserspuren zu bestimmen, so müßten Eichkurven mit möglichst vielen Meßpunkten u.a. im Bereich kleiner Wasserkonzentrationen aufgestellt werden. Dies stellt aber ein erhebliches Handicap für die praktische Anwendung dar.

In einer vorangehenden Arbeit [5] ist es gelungen, die Lösungsmittelpolarität binärer Gemische als Funktion ihrer Zusammensetzung in geschlossener Form darzustellen. (Die Vielzahl und Verschiedenartigkeit der untersuchten Gemische legt nahe, daß die gefundene Formel auf sehr viele binäre Gemische angewendet werden kann). Als Maß für die Lösungsmittelpolarität läßt sich der $E_{T} 30$-Wert $(I)$ (in einem bestimmten organischen Lösungsmittel) verwenden, der von der Konzentration des enthaltenen Wassers $(c)$ entsprechend Gl. (1) abhängt.

$E_{T} 30=E_{D} \cdot \ln \left(\frac{c}{c^{*}}+1\right)+E_{T}^{0}$.

$E_{T}^{0}$ ist der $E_{T} 30$-Wert des reinen, wasserfreien Lösungsmittels. Die Werte $E_{D}$ und $c^{*}$ der zwei-ParameterGleichung (1) sind für das jeweilige Lösungsmittel charakteristisch und für eine Reihe häufig verwendeter Lösungsmittel in Tabelle 1 zusammengestellt. Sie können für weitere Lösungsmittel leicht bestimmt werden (siehe experimenteller Teil).

Aus Gl. (1) wird durch Umformen Gl. (2) erhalten,

$c=c^{*} \cdot \exp \left(E_{T} 30 / E_{D}-E_{T}^{0} / E_{D}\right)-c^{*}$

mit der der Wassergehalt eines Lösungsmittels berechnet wird.
Zur Bestimmung des Wassergehaltes wird wie folgt verfahren: Wenig 1 wird in dem betreffenden Lösungsmittel gelöst und das Absorptionsmaximum der Solvatochromiebande von 1 mit einem UV-Spektrometer oder visuell durch Vergleich mit einer Farbskala bestimmt. Über Gl. (3) wird der $E_{T} 30$-Wert der Lösung ermittelt.

$$
E_{T} 30=28590\left[\mathrm{kcal} \cdot \mathrm{nm} \cdot \mathrm{Mol}^{-1}\right] \lambda_{\max } .
$$

Durch Einsetzen dieses Wertes zusammen mit den Tabelle 1 entnommenen $E_{D^{-}}$und $c^{*}$-Werten in Gl. (2) wird $c$, die Konzentration an Wasser, berechnet. Für eine genaue Bestimmung von $c$ ist der logarithmische Zusammenhang zwischen $\lambda_{\max }^{-1}$ und $c$ günstig, da hierdurch über einen weiten Meßbereich der relative Meßfehler nahezu konstant ist. Dies ist für den Bereich kleiner Wasserkonzentrationen von besonderem Vorteil. Wie experimentell überprüft worden ist [5], behält Gl. (1) und damit auch die abgeleitete Gl. (2) über viele Konzentrationsdekaden ihre Gültigkeit und ermöglicht daher die Analyse über einen weiten Konzentrationsbereich. Bei hohen Wasserkonzentrationen $\left(c>20 \mathrm{Mol} \cdot 1^{-1}\right)$ kann in wenigen Sonderfällen die Funktion(1) in eine andere, analoge mit anderen $c^{*}$ und $E_{T}$-Werten übergehen.

Der Vorteil der oben angegebenen Methode ist, daß sie leicht und schnell ausgeführt werden kann und nur geringe Mengen Farbstoff benötigt. Sie ist universell anwendbar (mit Ausnahme von Säuren, wie Ameisensäure, die den Farbstoff protonieren [6]). Gleichung (2) kann auch auf andere solvatochrome Systeme angewendet werden. Bei 1 ist jedoch die Solvatochromie besonders stark [6] und die Meßgenauigkeit daher groß.

\section{Experimenteller Teil}

Die UV-Spektren wurden mit dem UV-Spektrometer DMR-21 der Firma Zeiss aufgenommen. Für eine überschlägige Bestimmung von $\lambda_{\max }$ von $l$ und damit des Wassergehaltes genügt der Farbvergleich der Lösung mit einer Farbskala, z.B. Lit. [4].

\section{Bestimmung des Wassergehaltes} eines Lösungsmittels

Eine $1 \mathrm{~cm}$-Glasküvette, mit einem Stopfen versehen, wird mit dem zu untersuchenden Lösungsmittel gefüllt (gegebenenfalls unter Feuchtigkeitsausschluß) und mit einer geringen Menge $(<5 \mathrm{mg}) /$ versetzt (in einigen Lösungsmitteln ist die Lösegeschwindigkeit von 1 gering). Das UV-Spektrum der Solvatochromiebande von $I$ wird bei $25^{\circ} \mathrm{C}$ $(0.7 \leq E \leq 1.0)$ aufgenommen und $\lambda_{\max }$ der Solvatochromiebande bestimmt. Eine exakte Bestimmung von $\lambda_{\max }$ kann nach der Mathiasschen Regel [1] vorgenommen werden. Der so erhaltene Wert von $\lambda_{\max }$ wird mit Formel (3) in den $E_{T} 30$-Wert umgerechnet, der mit Hilfe von Gl. (2) und Tabelle 1 den Wassergehalt ergibt. Für eine überschlägige Bestimmung des Wassergehaltes wird nicht einmal ein Spektrometer benötigt. $\lambda_{\max }$ wird über einen visuellen Farbvergleich der Lösung mit einer Farbskala (z. B. Lit. [4]) erhalten. Die weitere Auswertung erfolgt nach der oben beschriebenen Prozedur. 
Bestimmung der Parameter $E_{D}$ und $c^{*}$ von weiteren Lösungsmitteln

In einem $10 \mathrm{ml}-\mathrm{Me} ß k$ olben werden bei definierter Temperatur 0,$5 ; 1$; $2 \ldots 9 \mathrm{ml}$ Wasser einpipettiert und bei $25^{\circ} \mathrm{C}$ mit dem betreffenden Lösungsmittel aufgefüllt. Die $E_{T} 30$-Werte dieser Lösungen werden nach dem oben angegebenen Verfahren bei $25^{\circ} \mathrm{C}$ bestimmt und der Wassergehalt in $c$ umgerechnet. In einem Diagramm wird $E_{T} 30$ gegen $\ln c$ aufgetragen und die Steigung $\left(E_{D}\right)$ und der Ordinatenabschnitt (b) des linearen Teils bestimmt. $c^{*}$ wird nach Formel (4) berechnet.

$c^{*}=\exp \left[\left(E_{T}^{0}-b\right) / E_{D}\right]$.

Für diese Prozedur steht das Rechenprogramm POLAR ${ }^{1}$ (in FORTRAN V) zur Verfügung, das auch die Meßwerte im nichtlinearen Teil der beschriebenen Kurve berücksichtigt. Die Werte $c^{*}$

1 Auf Wunsch wird das Rechenprogramm zur Verfügung gestellt und $E_{D}$ von Tabelle 1 wurden mit dem Rechenprogramm am Rechenzentrum der Universität Freiburg berechnet.

Herrn Prof. Dr. C. Rüchardt wird für die Unterstützung gedankt.

\section{Literatur}

1. Brdicka, R.: Grundlagen der Physikalischen Chemie, 10. Aufl., S. 252, 503. Berlin: VEB 1972

2. Dimroth, K., Reichardt, C.: Fresenius Z. Anal. Chem. 215, 344 (1966)

3. Dimroth, K., Reichardt, C., Siepmann, T., Bohlmann, F.: Liebigs Ann. Chem. 661, 1 (1963)

4. Klessinger, M.: Chemie in unserer Zeit 12, 1 (1978)

5. Langhals, H.: Nouv. J. Chim. (im Druck)

6. Reichardt, C.: Angew. Chem. 91, 119 (1979)

7. Reichardt, C., Dimroth, K. : Fortschr. Chem. Forsch. 11, 1 (1968)

8. Vogel, A. I.: A Textbook of quantitative inorganic Analysis, 4. Aufl., p. 688. London: Longman 1978

Eingegangen am 24. Juli 1980 